\title{
Partizipation als Ausnahmezustand
}

\section{Benedikt Korf, Zürich}

\section{Einführung}

Subpolitik, schreibt Ulrich BecK in «Die Erfindung des Politischen» (1993), ist der Ort, an dem die Grenze zwischen Politik und Nichtpolitik verschwindet. Die Orte der Entscheidung sind nicht mehr in den politischen Institutionen zu finden:

«Subpolitik unterscheidet sich von Politik [dadurch], dass (...) auch Akteure außerhalb des politischen und korporatistischen Systems auf der Bühne der Gesellschaftsgestaltung auftreten» (BECK 1993: 162).

In ihrer Entgrenzung durchdringt Subpolitik das hierarchische System der institutionellen Politik und hinterfragt es, schränkt seine Handlungsmöglichkeiten ein. Sie unterminiert damit den in der Moderne verankerten Glauben an das politische System (Parlament, Regierung) als exklusives Zentrum der Politik:

«Politik ist nicht länger der Ort oder auch nur der zentrale Ort, an dem über die Gestaltung der gesellschaftlichen Zukunft entschieden wird» (BECK 1986: 371).

Für BECK ist die gesellschaftliche Formierung von Subpolitik Anzeichen einer neuen alltagspraktischen politischen Kultur einer reflexiven Demokratisierung.

Ulrich BeCK denkt Subpolitik als eine aus dem Untergrund der Lebenswelt entstehende Politik. Doch sind Planer und Politiker darangegangen, diese Subpolitik zu institutionalisieren, also in die institutionalisierte Politik zu integrieren. Dies geschieht in der Form von Bürgerforen, Expertenhearings, Runden Tischen und anderen alternativen Formen der Bürgerbeteiligung, die nicht Teil der gesetzlich geregelten Entscheidungsfindungsprozesse sind, aber teilweise in diese kooptiert werden. So werden zum Beispiel regionale Förderprogramme der EU oft nicht über die institutionalisierte Politik abgewickelt, sondern durch alternative Runde Tische und Bürgerforen, die über die Verwendung von Fördermitteln entscheiden (MüLLER et al. 2002). Diese institutionalisierte Subpolitik als Entscheidungsverfahren ist deshalb zu unterscheiden von UlRICH BECK's Verständnis der Subpolitik als Entgrenzung des Politischen, das Partizipation als gesellschaftlich aktiv sein in Form des «sich Beteiligens» versteht (vgl. GeISER 2001).

In der Entwicklungszusammenarbeit (EZA) kann ein Trend festgestellt werden, der dieser Bürokratisierung von Subpolitik gleichkommt, ohne dass explizit auf BEcK's Schriften Bezug genommen würde (die dieser aus seiner soziologischen Analyse reflexiver Modernisierung in den demokratischen Wohlstandsgesellschaf- ten Europas abgeleitet und nicht auf Entwicklungsprobleme in Afrika oder Asien ausgedehnt hat). In der EZA zeigt sich die Bürokratisierung von Subpolitik in der Popularisierung partizipativer Methoden in den 1990er Jahren. Unter diesen Ansätzen ist Participatory Rural Appraisal (PRA) besonders erfolgreich gewesen (Chambers 1994), aber auch kritisiert worden.

Aus der zunehmenden Wirkmacht bürokratisierter Subpolitik ergibt sich die grundlegende Frage, mit welchem «Recht» und mit welcher «Legitimation» diese Art von Subpolitik in die Sphäre der institutionalisierten Politik eindringt und ihr Grenzen setzt (JAIN 1998: 59), sei es im Rahmen von europäischer Regionalförderung oder der EZA. In beiden Fällen wird die Legitimationsfrage in solchen Formen bürokratisierter Subpolitik besonders virulent, da diese Verfahren zu Entscheidungen über die Allokation signifikanter finanzieller Mittel führen. In diesem Beitrag wird in Anlehnung an Chantal Mouffe (2005) und Giorgio Agamben (2004) argumentiert, dass «Partizipation» (als bürokratisierte Subpolitik) eine Form von PostPolitik darstellt, die die Unterscheidung von Freund und Feind - und damit eine fundamentale ontologische Kategorie des Politischen - aufweicht, indem sie den Konsensus zur zentralen Logik des Politischen macht. Konsens wird zum Ausnahmezustand, in dem die «Norm», die «Ordnung» und die «Gesetze» - also das, was als die institutionalisierte Politik (die staatliche Ordnung) angesehen wird - zwar bestehen (bleiben), aber nicht zur Anwendung kommen. Andere Handlungen, hier die Konsensbildung, die nicht den Stellenwert von Gesetzen oder traditionellen Normen besitzt, gewinnen deren Kraft und ersetzen sie temporär. PRA kreiert solche Ausnahmezustände, die durch EZA zur Permanenz werden und damit die Unterscheidbarkeit zwischen Ordnung und Ausnahme - hier die EZA-Intervention - verwischen.

\section{Participatory Rural Appraisal (PRA) als bürokratisierte Subpolitik}

PRA ist eine Methode, ein Ansatz und eine Planungsphilosophie, die in der EZA in den letzten fünfzehn Jahren grosse Resonanz gefunden hat. Es ging, wie Robert Chambers, ein Hauptprotagonist von PRA, einst formulierte, um die Übergabe der Verantwortung und der Entscheidungsmacht an die Betroffenen selber. Chambers nannte dies handing over the stick (CHAmbers 1994). Planer und Aktivisten sollten moderieren und Ermöglichungsräume aufzeigen statt selbst zu planen. 
PRA wird meist in einem raum-zeitlichen «Container» aufgeführt, in Form eines zeitlich und räumlich geschlossenen Workshops, meist in mehrtägigen Veranstaltungen, an denen die Bevölkerung eines ganzen Dorfes beteiligt wird. Neben Veranstaltungen für die ganze Bevölkerung werden auch Sitzungen mit einzelnen sozialen Gruppen abgehalten, um deren spezifische Sicht aufnehmen und in die Plenumsveranstaltungen einbringen zu können. Externe Planer sollen als Moderatoren die gleichberechtigte Teilnahme aller Beteiligten durch spezielle Visualisierungsund Moderationsmethoden gewährleisten - die so genannte PRA-Toolbox. Am Ende dieses Verfahrens steht eine Problemanalyse mit Umsetzungsplänen, die von der ganzen Dorfgemeinschaft getragen und unterstützt wird. Dies, so eine der impliziten Annahmen von PRA, soll die ownership der Betroffenen an Projekten erhöhen, da diese Projekte als eigene wahrgenommen werden. Dadurch erhofft man sich eine höhere Nachhaltigkeit von Projekten, eine größere Beteiligung an der Umsetzung und eine bessere Angepasstheit an die lokalen sozialen und ökonomischen Rahmenbedingungen.

PRA kann man als eine Bürokratisierung von Subpolitik bezeichnen. PRA möchte eine Verschiebung der gesellschaftlichen Machtbereiche ermöglichen und EZA aus den politischen Institutionen des postkolonialen Staates und der bestehenden hierarchischen kulturellen Ordnungen herausführen - hin zu gesellschaftlichen Gruppen und Bewegungen der «Zivilgesellschaft» (vgl. GEISER 2007). PRA wurde in den 1990er Jahren bei vielen EZA-Organisationen populär, da es als eine effektive Antwort auf eine wenig erfolgreiche regionale Entwicklungsplanung der letzten zwanzig Jahre gesehen wurde (CHAMBERs 1994). PRA sollte helfen, Entwicklungsprojekte näher an den Bedürfnissen der Betroffenen zu planen. Durch Partizipation sollte die ländliche Bevölkerung politisch aktiviert werden, eigene Rechte einfordern und auf ihre Bedürfnisse hinweisen können (NELSON \& WRIGHT 1995).

\section{Partizipation als Tyrannei?}

In die allgemeine Euphorie, die mit dem PRA-Boom verbunden war, platzte 2001 ein Buch mit dem Titel «Participation - The New Tyranny?», das Theorie und Praxis von PRA einer grundlegenden Kritik unterzog (CoOKe \& Kothari 2001). Cooke und Kothari (2001) betrachteten PRA (und andere in der EZA praktizierte Partizipationsmoden und -diskurse) als illegitime, ungerechte Ausübung von Macht. Dies bezeichneten sie als Tyrannei, die sich in drei Weisen formiere (COOKE \& KOTHARI 2001: 7-8): erstens argumentierten sie, dass die Macht externer Moderatoren, die Entscheidungsfindungsprozesse oft in ihrem Sinne beeinflussten und kanalisierten, zu einer Tyrannei der Entscheidungsfindung führen könnten. Zweitens führe interner Gruppenzwang oft zu einer Tyrannei der Gruppe, mit der Folge, dass die Interessen der Mächtigen und Einflussreichen perpetuiert würden. Drittens seien viele PRA-Verfahren durch die mechanistische Anwendung des ausgefeilten Methodenbaukastens der PRA-Methode geprägt. Dies führe zu einer Tyrannei der Methode, da dann ein enges Methodenkorsett entstehe, das die Artikulation lokaler Bedürfnisse nur unzureichend anrege.

Tatsächlich sind diese Kritikpunkte sowohl in der Theorie (KAPOOR 2002, 2005; LEEUWIS 2000; WiLLIAMS 2004) als auch in der Praxis (AlfF et al. 1998; Korf 2002; KÜHL 1998; RAUCH 1996) detailliert bestätigt worden. In der Umsetzung führte die schnelle Verbreitung der Methode oft zu fragwürdigen Praktiken. So zeigte sich zum Beispiel, dass es vielen Entwicklungsexperten, NGO-Aktivisten und lokalen Bürokraten schwer fiel, ihre eigene Rolle umzudeuten und ihre kulturelle Überheblichkeit, die sich aufgrund von Kasten- oder Klassenzugehörigkeit in ihren alltäglichen Habitus eingeschlichen hatte, zu überwinden. Auch zeitigten die Prozesse und Ergebnisse vieler PRA-Workshops nur wieder konventionelle Projektvorschläge und shopping lists, die sich an dem orientierten, was die lokale Bevölkerung mit «Entwicklung» in Verbindung brachte, also dem, was ihr von lokalen Bürokraten und NGO-Aktivisten seit langem suggeriert wurde. In anderen Fällen wurde PRA ein Verfahren zur Legitimierung von Elitenentscheidungen.

Ein grundlegendes Problem stellte sich mit der Frage der Breitenwirksamkeit. Sollte PRA auf einzelne «Projektinseln» beschränkt, also eine Ausnahmeerscheinung bleiben? Oder sollte es eine Standardmethode der ländlichen Entwicklungsplanung werden und damit möglichst vielen Menschen und Gemeinden zugute kommen? Hier eröffnet sich ein Spannungsfeld, denn eine auf Ermächtigung abzielende Partizipationsmethode, als die sich PRA insbesondere in ihrer Gründungsperiode verstand, lässt sich schwerlich als ein staatliches Planungsverfahren verordnen, durch das eben diese bürokratische Ordnung und ihre Macht potentiell herausgefordert würde. Nichts anderes würde Ermächtigung bislang Stimmloser und deren Teilhabe an der Macht letztendlich bedeuten. Insgesamt bleibt deshalb die Bilanz von PRA ambivalent: zwar haben die vielfältigen PRA-Prozesse auf lokaler Ebene erst die Notwendigkeit und die Möglichkeit demonstriert, die lokale Bevölkerung an Entwicklung zu beteiligen (Hickey \& MoHan 2004; Williams 2004), doch haben sich in den Niederungen der Praxis auch die Grenzen von PRA gezeigt. 


\section{Das Paradox der Entscheidungsfindung in PRA}

Das Kernproblem, auf das der Tyranneivorwurf hinausläuft, ist die Legitimationsfrage: Wie kann man beurteilen, ob und wann eine Entscheidung aus einem PRA-Prozess legitim (bzw. legitimiert) ist? Zwei Gründe sprechen dafür, dass sich ein PRA-Verfahren einer besonders kritischen Prüfung stellen muss. Erstens ist PRA ein Ausnahmeverfahren, das bestehende, institutionalisierte Entscheidungsverfahren ersetzt. Konventionellen Gremien, die in institutionalisierten Verfahren zur Anwendung kommen, zum Beispiel das Gemeindeparlament in einem Dorf in Brandenburg oder der Ältestenrat eines Chiefs in Ghana, besitzen bereits eine Legitimität zur Entscheidungsfindung. Im Falle demokratischer Parlamente wird die rechtfertigende Macht über die soziale Praxis von (Wahl-)Verfahren - eingebettet in eine verfassungsrechtliche Ordnung - erbracht. Im Falle traditioneller Autoritäten, wie eines ghanaischen Chiefs, wird diese Legitimität genealogisch aufgrund familiärer Herkunft abgeleitet.

Dass diese Verfahren bereits bestehen und praktiziert werden, sagt noch nichts über ihre Gerechtigkeit oder Angemessenheit aus. Doch beziehen sie faktische Legitimation durch die sozialen Praktiken der Herrschaftsausübung und ihrer Verfahren. PRA hingegen kommt in dieses politische Feld als etwas, das bislang noch nicht vorhanden war, als ein externer Verfahrensvorschlag zur Generierung von Legitimation kollektiver Entscheidungen. PRA muss deshalb die Quellen seiner Legitimation explizit und gegenüber den vorhandenen Verfahren als überlegen ausweisen. Dieser Legitimationsdruck wird noch durch den Tatbestand verschärft, dass PRA oft als Verfahren angewendet wird, um substantielle finanzielle Ressourcen der EZA in den ländlichen Raum zu kanalisieren, die die Entwicklung ländlicher Kommunen signifikant beeinflussen.

Legitimation kann in einem Verfahren entweder über den Prozess oder das Resultat hergestellt werden. Da Subpolitik oft in einem Kontext stattfindet, in dem immer weniger Einigkeit über «Fakten» und Ergebnisse der Politik erzielt werden kann, gewinnen prozedurale und diskursive Rationalität an Bedeutung (CONRAD 1997). Legitimation wird dann durch Verfahren ausgewiesen. PRA stellt Legitimität durch Konsens her. PRA ist als ein offenes Forum konzipiert, in dem potentiell alle Betroffenen in die Problemanalyse und Entscheidungsfindung eingebunden werden. Grundidee des offenen Forums ist, dass Deliberation, verstanden als der herrschaftsfreie Diskurs gleichberechtigter Diskurspartner und -partnerinnen, zu einem Konsens führt - also zu einer Entscheidung (über das, was die zentralen Probleme sind, und mit welchen
Aktionen sie überwunden werden können). Entscheidungsfindung im Konsens wird über die Praktik des herrschaftsfreien Diskurses legitimiert, entsprechend des diskursethischen Konzeptes von JÜrgen HABERmas (HABERmas 1991), obwohl dieser Bezug in der PRA-Literatur kaum erklärt wird (vgl. kritisch dazu KAPOOR 2002; LeEuwIS 2000: 937). Für Habermas ist der herrschaftsfreie Diskurs, also der Prozess der Deliberation, ausschlaggebend für die Gerechtigkeit und Legitimität eines Verfahrens und damit auch seines Ergebnisses (Habermas 1994).

Implizit liegt bei PRA jedoch ein Paradox in der Entscheidungsfindung vor: PRA verinnerlicht zwei Logiken, die sich widersprechen. Es sind dies die Kant'sche und die Hegel'sche Logik (vgl. Korf 2007: 264). Auf der einen Seite soll PRA gerade die lokal verdichteten Bedürfnisse, Erfahrungen und Deutungen in ihrer historischen Gewordenheit «zur Sprache bringen» - es geht gerade nicht um die Überstülpung westlicher Vorstellungen und Vorschläge. Dies soll hier die Hegel'sche Logik genannt werden, da sie sich auf ein lokales, historisch gewordenes, kollektives Subjekt («die Dorfgemeinschaft») bezieht. Dieses historisch gewordene kollektive Subjekt wird jedoch durch das Ideal einer universalistischen Methode konstituiert, einer Art KANT'schen Logik. Das Spannungsfeld ergibt sich daraus, dass auf der einen Seite mit einer partikularistischen Logik der Legitimität argumentiert wird, aber dafür ein universalistisch begründetes Verfahren (Diskursethik) in Anwendung gebracht wird.

Schon für sich genommen weisen beide Logiken grundlegende Probleme auf (vgl. Abbildung 1): Im Falle der HegeL'schen Logik handelt es sich um einen naturalistischen Fehlschluss, da aus der historischen Gewordenheit auch die normative Gewünschtheit abgeleitet wird. Doch lässt sich aus einem Ist-Zustand nicht zwingend dessen Normativität ableiten - nicht alles, was ist, sollte auch sein. So rügte schon DAVID Hume im dritten Buch seines «Treatise of Human Nature» (1978 [1740], Book III,I.1) den unvermittelten Übergang von beschreibenden Sätzen, die eine Tatsache feststellen, zu solchen, die eine Soll-Vorschrift machen. Hierfür seien Gründe anzugeben, die nicht schon mit einem Faktum allein gegeben sind (in diesem Fall die historische Gewordenheit). Entscheidungen, die sich ein historisch gewordenes kollektives Subjekt (eine Dorfgemeinschaft) gibt, müssen deshalb nicht notwendigerweise gerecht und legitim sein, nur weil sie aus einem historisch gewordenen Legitimationsverfahren, z.B. der genealogisch gerechtfertigten Häuptlingsherrschaft, entstanden sind.

Im Falle der KanT'schen Logik zeigt sich das Problem der deliberativen Lücke: Das Konzept des herrschaftsfreien Diskurses ist ein normatives Ideal. In der 


\begin{tabular}{|l|c|}
\hline \multicolumn{1}{|c|}{ Hegel'sche Logik } & \multicolumn{1}{c|}{ Kant'sche Logik } \\
\hline $\begin{array}{l}\text { Partikularistische Logik der } \\
\text { historischen Gewordenheit } \\
\text { des kollektiven Subjekts }\end{array}$ & $\begin{array}{l}\text { Universalistisches Ideal des } \\
\text { herrschaftsfreien Diskurses }\end{array}$ \\
Gefahr: naturalistischer Fehlschluss & Gefahr: deliberative Lücke \\
\hline
\end{tabular}

Abb. 1: Spannungsfeld von Hegel'scher und Kant'scher Logik

Conflicting rationales of Hegelian and Kantian logics

Tension selon la logique hégélienne et kantienne

realen Welt kann dieses Ideal angestrebt werden, aber praktische politische Diskussionen werden nicht nur durch den Austausch rational begründeter Argumente geprägt, sondern auch durch Emotionen, Einschüchterungen, Demagogie und Begeisterungsfähigkeit (WALzer 1999). Das Konzept von PRA sieht im Moderator und in den Visualisierungsmethoden die Instrumente, um sich an das Ideal eines herrschaftsfreien Diskurses anzunähern (KAPOOR 2002; LEEUWIS 2000). Doch wer setzt die Agenda? Wer beteiligt sich am Diskurs? Wer kann sich überhaupt die Zeit nehmen, an einem Workshop teilzunehmen (vgl. Agarwal 2001; Korf 2002; Mosse 2001)? Die PRA-Instrumente reichen offensichtlich nicht aus, einen herrschaftsfreien Diskurs herzustellen (CoOKe \& Kothari 2001; LeeuWIS 2000; KAPOOR 2002). Auch stellt sich die Frage, ob das Konzept des herrschaftsfreien, offenen Diskurses in allen Gesellschaften als angemessene Methode der Entscheidungsfindung anerkannt ist, oder ob es sich hier um einen Eurozentrismus handelt, der ein im Westen anerkanntes, normatives Prinzip universalisiert (vgl. KAPOOR 2002).

\section{Partizipation als post-politischer Ausnahmezustand?}

In ihrer Streitschrift «On the Political» (2005) hat Chantal Mouffe die Problematik einer konsensusorientierten, auf Deliberation abzielenden Subpolitik als post-politische Aspiration kritisiert. ChantaL MOUfFe nimmt insbesondere UlRICH BECK's Beobachtungen zur Neuorientierung des Politischen ins Visier. Für BECK ergibt sich aus der zunehmenden Individualisierung der Gesellschaft die Möglichkeit, das Politische neu zu erfinden, und zwar als eine Politik, die sich vom Entweder-oder-Schema (entweder links oder rechts, entweder Kapitalismus oder Sozialismus) verabschiedet (BeCK 1993). Diese Neuerfindung des Politischen findet sich für BECK in der Subpolitik, die sich außerhalb der institutionalisierten Politik abspielt. CHANTAL
MoufFe sieht in BECK's Vorschlägen eine gefährliche Tendenz zur Post-Politik. Post-politisch deshalb, weil ULRICH BECK aus ihrer Sicht die grundlegende ontologische Dimension des Politischen verkennt: den Antagonismus der Freund-Feind-Unterscheidung.

Mouffe beruft sich auf das klassische Argument von CARL Schmitt, der schon 1922 argumentierte, dass man Politik nicht ohne den Feind denken könne (SchmiтT (2004 [1922]). Für Mouffe besteht in der post-politischen Aspiration die Gefahr, dass durch das Bedürfnis nach rationaler Konsensus-Bildung die Freund-FeindUnterscheidung tabuisiert werde, die das Politische ontologisch begründe. Nur über Unterscheidungen von «wir» und «sie» sei Identitätsbildung möglich. Durch post-politische Konsensverfahren werde diese Unterscheidung unterlaufen, doch könne man das Denken im Freund-Feind-Schema letztlich nicht verhindern. In der post-politischen Aspiration würden, so MoufFe, moralisierende Freund-Feind-Unterscheidungen getroffen, die zum Ausschluss bestimmter Gruppen führten und dadurch erst zu verschärften gesellschaftlichen Antagonismen beitrügen, was sich zum Beispiel im Anwachsen des Rechtspopulismus in Europa zeige (Mouffe 2005: 69f.). Stattdessen sei es notwendig, die Freund-Feind-Unterscheidung zuzulassen und durch agonistische Verfahren zu bündeln und zu kanalisieren. Chantal Mouffe plädiert deshalb für eine «kämpferische» Politik, die die agonistische Auseinandersetzung zwischen «uns» und «ihnen» zulässt, jedoch in gezähmte Bahnen lenkt.

Inwiefern ist auch PRA eine post-politische Aspiration? PRA ist eine Form bürokratisierter Subpolitik, ein Verfahren, das konsensbildende Entscheidungsfindung in der EZA institutionalisieren möchte. Damit wird «Entwicklung» und «EZA» aus dem Bereich der Politik und über das Politische hinaus gehoben. PRA soll Politik als schmutziges Geschäft aus der EZA heraushalten. Die verschiedenen Instrumente und Prin- 


\begin{tabular}{|l|c|}
\hline \multicolumn{1}{|c|}{ Carl Schmitt } & Giorgio Agamben \\
\hline $\mathrm{AZ}=$ Ereignis (des Rechts) & $\mathrm{AZ}=$ topologische Figur \\
Dezision (Entscheidung) & Ununterscheidbarkeit \\
\hline
\end{tabular}

\begin{abstract}
Abb. 2: Ausnahmezustand (AZ)
State of exception

Etat d'exception
\end{abstract}

zipien (Moderation, Visualisierung), die in PRA zur Anwendung kommen, sollen Entwicklung als einen Prozess aus dem Freund-Feind-Schema lokaler und regionaler Antagonismen herausnehmen. PRA schafft einen politischen Ausnahmezustand, indem es einen politischen Raum jenseits der institutionalisierten Politik eröffnet. PRA ist insofern «politisch» im Sinne der «Post-Politik», und damit gerade nicht das, was Carl Schmitt oder Chantal Mouffe als «das Politische» bezeichnen.

PRA als Post-Politik schafft somit einen Ausnahmezustand, einen Zustand außerhalb der institutionalisierten Politik. Der Begriff «Ausnahme», oder genauer «Ausnahmezustand», wurde ursprünglich von CARL Sснмітт mit dem Diktum «Souverän ist, wer über den Ausnahmezustand entscheidet» (SснмIтT [1922] 2004: 1) geprägt. Für Schмiтt wird das Recht im Ausnahmezustand begründet, als nicht begründbar, sondern nur aufgrund einer Entscheidung geltungsfähig. Was als letzte Maßnahme der staatlichen Ordnung erscheint, nämlich die Option, die gesamte Rechtsordnung aufzuheben, markiert für ScHмITT den Ursprung aller Rechtsgeltung. Die Ausnahme ist in der Entscheidung über den Ausnahmezustand begründet. CARL SCHMITT war fasziniert von der Entscheidung als rechtsetzende Kraft im Ausnahmezustand. In diesem Sinne könnte man PRA als einen raum-zeitlichen «Container» des Ausnahmezustands ansehen, der durch die dezisionistische Dynamik des Verfahrens zur rechtssetzenden Kraft wird.

In Abgrenzung zu Carl Schmitt betont Giorgio Agamben hingegen die Ununterscheidbarkeit von Ausnahmezustand und Regel (vgl. Abbildung 2): der Ausnahmezustand wird zur Regel. Grorgio Agamben definiert den Ausnahmezustand als

«(...) einen Zustand des Gesetzes, in dem die Norm zwar gilt, aber nicht angewendet wird (weil sie keine 〈Kraft〉 hat) und auf der anderen Seite Handlungen, die nicht den Stellenwert von Gesetzen haben, deren Kraft gewinnen» (Agamben 2004: 49).
Agamben formuliert hier das Paradox, dass im Ausnahmezustand eine Ineffektivität des Gesetzes wirksam ist - eine passive Aktivität, eine Wirkung ohne Wirkkraft. Während für Schmirt der Ausnahmezustand als Ereignis des Rechts noch im Recht verortet ist, verwischen sich für AGAMBEN Gewalt und Gerechtigkeit im Inneren des Rechts. Für Agamben ist der Ausnahmezustand eine topologische Figur und durchdringt das Recht:

«Der Ausnahmezustand ist demnach nicht so sehr eine raumzeitliche Aufhebung als vielmehr eine topologische Figur, in der nicht nur Ausnahme und Regel, sondern Naturzustand und Recht, das Draussen und das Drinnen, ineinander übergehen» (Agamben 1998: 48).

Konsens, PRA und «Partizipation» sind, was GIorgio Agamben als die «Schwelle der Ordnung» bezeichnet - der Ursprung, der Ort, an dem die Konstitution der Ordnung gemacht wird. PRA ist insofern ein Ausnahmezustand, als es eine Maßnahme ist, die Ordnung (die institutionalisierte Politik) aufzuheben, temporär außer Kraft zu setzen. So werden Projekte nicht mehr im Ältestenrat eines ghanaischen Chiefs, in der Runde der Klanältesten in Somalia oder den Prozeduren eines Gemeindeparlaments in Deutschland verhandelt, sondern im politischen Raum der Deliberation, der durch PRA hergestellt wird. Zwar können in diesem Raum auch die Akteure der konventionellen Ordnung, zum Beispiel der Chief, der Bürgermeister oder der Gemeinderat teilnehmen, doch werden die Verfahrensregeln und der Prozess von auswärtigen Akteuren, den PRA-Moderatoren, gesteuert.

Somit wird PRA zum Ort, an dem sich die Ordnung mit dem berührt, was nicht mehr zur Ordnung gehört. PRA ist der Ort, an dem sich «lokale» Gesellschaften und ihre Ordnung mit der Welt der EZA treffen. Doch zeigt sich in der Entwicklungspraxis, dass PRA nicht ein temporärer Ausnahmezustand bleibt, eine einmalige Projektinsel. PRA wird permanent, immer wieder durch- und aufgeführt. Durch PRA wird eine neue Ordnung konstituiert, der permanente Ausnahmezu- 
stand der EZA, der viele Empfängerländer in Afrika und Asien durchdringt und traditionelle Autoritäten und Verfahren unterminiert. PRA als Ausnahme bleibt nicht auf ein temporäres Ereignis beschränkt, das einmalig die bestehende Ordnung aufhebt (und sie danach wieder einsetzt). PRA wird zur topologischen Figur, zur Beziehungsform (vgl. Geulen 2005: 73). PRA als permanente Ausnahme verwischt die Freund-Feind-Schemata der bestehenden Ordnung - und verhindert so die agonistische Auseinandersetzung, die Chantal Mouffe für den grundlegenden Bestandteil des Politischen hält.

Freilich gibt es in vielen Einsatzgebieten der EZA neben den traditionellen (z.B. Chief), den postkolonialen, staatlichen (z.B. gewählte Dorfparlamente) und den neuen, technokratischen Entscheidungsforen (z.B. PRA) auch lokale Formen von Entscheidungsfindung, die gewaltorientiert sind und sich in gewaltoffenen Räumen (ELWERT 1997) von Bürgerkriegen oder im Kontext von Bandenkriminalität entwickeln. Diese schaffen ganz andere «Ausnahmezustände» als die EZA. Auch in diesen gewaltoffenen Räumen wird die bestehende Ordnung suspendiert - doch nicht durch eine Aufweichung des Freund-Feind-Schemas, sondern durch dessen gewaltsame Instrumentalisierung.

\section{Schlussfolgerungen}

In der achten These über den Begriff der Geschichte schreibt Walter Benjamin, dass «(...) der Ausnahmezustand, in dem wir leben, die Regel ist» (BENJAMIN 1965: 84). Benjamin erkennt im Bestehenden selbst das Monopol der Legalität, der Wahrheit, des Rechts, dessen Gewaltcharakter sich in den «Ausnahmezuständen» (der Weimarer Republik und des Dritten Reiches) zeigte. In gewisser Weise versucht auch die EZA mit ihrem «Will to Improve» (Li 2007) einen Ausnahmezustand herbeizuführen, der über die legalistische oder genealogische Konstruktion von Legitimation und Recht in postkolonialen Staaten mit ihren hybriden Herrschaftsstrukturen hinausgeht. EZA als bürokratisierte Subpolitik postuliert damit neue Räume für das Politische.

Walter Benjamin geht es um die Herbeiführung des wirklichen Ausnahmezustandes als eines revolutionären Aktes (MARCuSE 1965: 100), nicht als ein technokratischer Akt bürokratisierter Subpolitik. PRA und EZA sind nicht revolutionär, sondern vermischen sich mit der bestehenden Ordnung. PRA wird somit nicht zum wirklichen Ausnahmezustand, sondern zum Ort, an dem die bestehende Ordnung temporär suspendiert wird. Doch führt die Logik der EZA zu einem Ausnahmezustand in Permanenz, wodurch das Innen und Außen der bestehenden Ordnung verwischt wird, also das, was traditionelle Autoritäten, postkoloniale, staatliche Strukturen und die Welt der EZA unterscheidet. Ausnahmezustand und Regel werden ununterscheidbar. Partizipation als Ausnahmezustand wird zur Regel.

PRA schafft Räume der Entscheidungsfindung außerhalb der bestehenden Ordnung, doch bleiben diese Räume an die bestehende Ordnung rückgebunden - die Ausnahme wird permanent - als EZA und vermischt sich mit der bestehenden Ordnung. Die «Ordnung» bleibt machtlos, jedoch präsent. Sie bleibt anwesend, jedoch in Nicht-Anwendung, lediglich als Potentialität. EZA generiert den Ausnahmezustand, der dann zum Ursprung einer bürokratisierten Ordnung wird, die Ausnahme und Regel vermischt. EZA und Nicht-EZA-Welt werden ununterscheidbar, jedoch in einer Welt, in der «das Politische» verloren gegangen ist.

Diesem Aufsatz liegt die Prämisse zugrunde, dass EZA nicht prima facie legitimiert ist, das Politische aus den Entwicklungsrealitäten postkolonialer Orte zugunsten einer kosmopolitisch moderierten Konsensordnung zu vertreiben. «Entwicklung» ist politisch, nicht post-politisch. Für die EZA bedeutet dies, Entwicklung nicht in raum-zeitlichen «Containern» von Ausnahmezuständen bürokratisierter Subpolitik zu kreieren, sondern das Politische in der Entwicklung zuzulassen, sich auf das Politische «in» der Politik, an den Orten der Politik, im politischen «System» einzulassen.

\section{Literatur}

Agamben, G. (1998): Homo Sacer. - Frankfurt am Main: Suhrkamp.

Agamben, G. (2004): Ausnahmezustand. - Frankfurt am Main: Suhrkamp.

Agarwal, B. (2001): Participatory exclusions, community forestry and gender: an analysis for South Asia and a conceptual framework. - In: World Development 29, 10: 1623-48.

Alff, U., Ay, P. \& E. Bauer (1998): Partizipation: mit offizieller Anerkennung ins Abseits. - In: Peripherie 72: 71-81.

BECK, U. (1986): Risikogesellschaft. - Frankfurt am Main: Suhrkamp.

Beck, U. (1993): Die Erfindung des Politischen. Frankfurt am Main: Suhrkamp.

BECK, U. (1997): The reinvention of politics. - London: Polity.

Benjamin, W. (1965): Zur Kritik der Gewalt und andere Aufsätze. - Frankfurt am Main: Suhrkamp.

Chambers, R. (1994): The origins and practice of Participatory Rural Appraisal. - In: World Development 22, 7: 953-969.

Conrad, J. (1997): Nachhaltige Entwicklung - ein 
ökologisch modernisiertes Modell der Moderne? - In: Brand, K.-W. (Hrsg.): Nachhaltige Entwicklung: eine Herausforderung an die Soziologie. - Opladen: Leske + Budrich: 51-69.

Cooke, B. \& U. Kothari (2001): Participation: the new tyranny? - London: Zed Books.

Elwert, G. (1997): Gewaltmärkte: Beobachtungen zur Zweckrationalität von Gewalt. - In: Kölner Zeitschrift für Soziologie und Sozialpsychologie, Sonderheft 37, 59-85.

GeIser, U. (2001): Reading «participation in forest management» through «modern» and «postmodern» concepts, or: where to start the normative debates? - In: Tovey, H. \& M. Blanc (eds): Food, nature and society. - Aldershot: Ashgate: 210-230.

Geiser, U. (2007): «Zivilgesellschaft» in Pakistan. Zur Schwierigkeit der Lokalisierung spezifischen sozialen Raums in fragilen postkolonialen Verhältnissen. - In: Asiatische Studien LXI, 4: 1183-1200.

Geulen, E. (2005): Giorgio Agamben zur Einführung. - Hamburg: Junius.

Habermas, J. (1991): Erläuterungen zur Diskursethik. - Frankfurt am Main: Suhrkamp.

Habermas, J. (1994): Faktizität und Geltung. - 4., erweiterte Auflage, Frankfurt am Main: Suhrkamp.

Hickey, S. \& G. MoHAN (2004): Participation: from tyranny to transformation? - Exploring new approaches to participation in development. - London: Zed Books. Hume, D. (1978 [1740]): A treatise of human nature. - Oxford: Oxford University Press.

JAIN, A.K. (1998): Subpolitik als Metapolitik. - In: HP - Zeitschrift der Historiker und Politologen an der Uni München 8: 56-59.

KAPOOR, I. (2002): The devil's in the theory: a critical assessment of Robert Chambers' work on participatory development. - In: Third World Quarterly 23, 1: 101-17.

KAPOOR, I. (2005): Participatory development, complicity and desire. - In: Third World Quarterly 26, 8: 1203-1220.

Korf, B. (2002): Ist PRA in der Postmoderne angekommen? - Peripherie 87: 293-314.

KorF, B. (2007): Governing bottom-up in rural development: the legitimacy dilemma. - In: CHeshire, L., Higgins, V. \& G. LAWRence (eds): Rural governance. - London: Routledge: 259-272.

KüHL, S. (1998): Wenn Partizipation zum Problem wird: die begrenzte Nützlichkeit von Partizipation in Entwicklungsprojekten - Erfahrungen aus Zentralafrika. - In: Peripherie 72: 51-70.

LEEUWIS, C. (2000): Reconceptualising participation for sustainable rural development: towards a negotiation approach. - In: Development and Change 31: 931-959. LI, T.M. (2007): The will to improve: governmentality, development and the practice of politics. - Durham, N.C.: Duke University Press.

Marcuse, H. (1965): Nachwort. - In: Benjamin, W.
(1965): Zur Kritik der Gewalt und andere Aufsätze. - Frankfurt am Main: Suhrkamp: 99-107.

Mosse, D. (2001): People's knowledge? Participation and patronage: operations and representations in rural development. - In: Cooke, B. \& U. Kothari (eds): Participation: the new tyranny? - London: Routledge: 1635 .

Mouffe, C. (2005): On the political. - London: Routledge.

Müller, K., Dosch, A., Morhbach, E., Aenis, T., Baranek, E., Boeckmann, T., Sieber, R. \& V. Toussaint (2002): Wissenschaft und Praxis der Landschaftsnutzung. - Weikersheim: Margraf.

Nelson, N. \& S. Wright (eds) (1995): Power and participatory development. - London: Intermediate Technology Publications.

RAUCH, T. (1996): Nun partizipiert mal schön. Modediskurse in den Niederungen entwicklungspolitischer Praxis. - In: blätter des iz3w 213: 20-22.

Schmitt, C. (2004 [1922]): Politische Theologie: vier Kapitel zur Lehre von der Souveränität. - 8. Auflage, Berlin: Dunkler \& Humblot.

WALzer, M. (1999): Vernunft, Politik und Leidenschaft. - Frankfurt am Main: Fischer.

Williams, G. (2004): Evaluating participatory development: tyranny, power and (re)politicisation. - In: Third World Quarterly 25, 3: 557-578.

\section{Zusammenfassung: Partizipation als Ausnahme- zustand}

In «Die Erfindung des Politischen» (1993) beschreibt Ulrich BeCK Subpolitik als den Ort, an dem die Grenze zwischen Politik und Nichtpolitik verschwindet. Aus der zunehmenden Wirkkraft von Subpolitik erwächst die grundlegende Frage, mit welcher Legitimation diese Art von Subpolitik in die Sphäre der institutionalisierten Politik eindringt. BECK's Subpolitik soll im Anschluss an theoretische Arbeiten von CHANTAL Mouffe und Giorgio Agamben als post-politische Aspiration bezeichnet werden, die einen Ausnahmezustand herstellt, indem sie Räume für das Politische postuliert und diese Ausnahmeräume zur Regel erklärt. Dieses Argument wird anhand einer bürokratisierten Form von Subpolitik analysiert, die in der Entwicklungszusammenarbeit (EZA) Eingang gefunden hat: der partizipativen Planungsmethode PRA.

Schlüsselwörter: Partizipation, Subpolitik, post-politisch, Ausnahmezustand, Legitimität

\section{Summary: Participation as state of exception}

In «The Reinvention of Politics» (1997), ULRICH BECK describes subpolitics as the space where the boundaries between politics and non-politics blur. However, the increasing leverage of subpolitics in reflexive modernity begs the question how such kind of subpoli- 
tics gain their legitimacy to permeate and substitute institutional forms of politics. In this paper, it is argued in line with Chantal Mouffe and Giorgio Agamben, that BECK's subpolitics may be seen as a post-political aspiration - an aspiration that produces a state of exception as it postulates a space for the political outside or beyond institutional politics, and declares this exception a rule. Empirical evidence is offered to support this view with an analysis of a bureaucratized form of subpolitics that has had a prominent place in development cooperation: the participatory planning method PRA.

Keywords: participation, subpolitics, post-political, state of exception, legitimacy

\section{Résumé: La participation comme état d'exception}

Dans «Die Erfindung des Politischen» (La réinvention du politique) (1993), Ulrich BeCK décrit la «subpolitique» comme l'espace dans lequel les frontières entre la politique et la non-politique se mélangent. Cependant, la force croissante de la subpolitique dans la réflexion portant sur la modernité soulève la question de savoir d'où provient la légitimité de cette subpolitique, qui lui permet d'infiltrer et de se substituer aux formes politiques institutionnelles. Dans le prolongement des travaux de Chantal Mouffe et Girogio
Agamben, cet article soutient l'idée selon laquelle la subpolitique de BECK n'est rien d'autre qu'une aspiration post-politique. Cette aspiration produit un état d'exception, dans la mesure où elle s'appuie sur un espace situé en dehors ou au-delà de la politique institutionnelle, et déclare cette exception comme étant la règle. Cet argument est examiné empiriquement au moyen d'une analyse d'une forme bureaucratique de subpolitique qui occupe une place prépondérante dans la coopération au développement: la méthode de planification participative PRA.

Mots-clés: participation, subpolitique, post-politique, état d'exception, légitimité

Prof. Dr. Benedikt Korf, Geographisches Institut, Universität Zürich, Winterthurerstrasse 190, CH-8057 Zürich, Schweiz.

e-mail: benedikt.korf@geo.uzh.ch

Manuskripteingang/received/manuscrit entré le 17.9.2008

Annahme zum Druck/accepted for publication/accepté pour l'impression: 20.5.2009 